\title{
Task Allocation Model for Optimal System Cost Using Fuzzy C-Means Clustering Technique in Distributed System
}

\author{
Seema Yadav ${ }^{1 *}$, Rakesh Mohan ${ }^{1}$, Pradeep Kumar Yadav ${ }^{2}$ \\ ${ }^{1}$ Department of Mathematics, DIT University, Dehradun 248009, India \\ ${ }^{2}$ CBRI-IIT Roorkee, Roorkee 247667, India
}

Corresponding Author Email: seema.yadav@ dituniversity.edu.in

https://doi.org/10.18280/isi. 250108

Received: 8 October 2019

Accepted: 10 December 2019

\section{Keywords:}

distributed system, task scheduling, load balancing, fuzzy c-means, Hungarian method

\begin{abstract}
The task scheduling is an important activity in distributed system environment to divide the proper load among the available processors. The requirement of efficient task scheduling technique is an important issue in distributed computing systems, which can balance the load in such a way, so that no processor remains idle. Further, it can provide proper utilization of available resources and minimize the response time and system cost, with the maximum system reliability. In this paper the novel task allocation technique is being proposed with the aim of minimizing the response time and system cost. The method of clustering is used for the proper distribution of tasks on the processors. The proposed technique uses Fuzzy C-Means clustering technique and Hungarian method for task allocations. The performance of the algorithm is evaluated through examples and the results are compared with some existing models.
\end{abstract}

\section{INTRODUCTION}

With advance computational technologies and high-speed networks, distributed computing system (DCS) has become popular worldwide. Distributed computing system has multiple processors located at geographically distant places i.e. at different cities or countries, interconnected by communication links. There are many factors which considerably affects the performance of the DCS viz. speed of processors, memories, failure rate of processors, failure rate of interconnecting network etc. One such \& highly considerable factor is allocation of modules to processors. This allocation should be in such a way that system cost is minimized with some average load on each processor, so that no processor remains idle. Also, the available resources should be utilized to its maximum. Task allocation can be done in two ways:

1. Static Allocation- when a module is assigned to a processor, it remains with the processor till the completion of the process.

2. Dynamic Allocation- a module when allocated to one processor may migrate to another processor according to requirement of the system.

Dynamic allocation uses current state information of the system in making decision while static allocation using Random or Round Robin don't use any information of current state of nodes for load balancing [1-4]. Different algorithms for module allocation are proposed with different objectives. Some have objective of balancing the load $[2,4]$ while some an objective of minimizing response time and maximizing system reliability [5-9]. Topcuoglu et al. [10] discussed and proposed two novel scheduling algorithms, the Heterogeneous Earliest-Finish-Time (HEFT) algorithm and the Critical-Pathon-a-Processor (CPOP) algorithm, for a bounded number of heterogeneous processors with an objective to meet high performance and fast scheduling time simultaneously. Falta et al. [11] propose a fully distributed K-Means algorithm (Epidemic K-Means) which does not require global communication and is intrinsically fault tolerant, which otherwise lacks in large scale systems and provides a clustering solution which can approximate the solution of an ideal centralized algorithm over the aggregated data as closely as desired. Rashidi [12] proposes an algorithm, based on multi-objective scheduling cuckoo optimization algorithm (MOSCOA), in which each cuckoo represents a scheduling solution in which the ordering of tasks and processors allocated to them are considered. In addition, the operators, of cuckoo optimization algorithm defined, are usable for scheduling scenario of the directed acyclic graph of the problem. Bahmani and Mueller [13] proposed a fast signaturebased clustering algorithm that clusters processes exhibiting similar execution behavior. Vidyarthi and Tripathi [14] developed a heuristic approach, based on genetic algorithm, to find the near optimal solution.

In this paper, the proposed work uses Fuzzy C-Means (FCM) clustering algorithm to allocate task to different processors with the objective of minimizing system cost and response time. It is different from other clustering techniques in such a way that the data point is not a member of only one cluster, but may belong to more clusters with certain degree of membership value. If the data points are located on the boundaries of the clusters, they are not forced to belong to a certain cluster and thus have flexibility of being the member of others clusters too, for better performance of system. FCM is an iterative process and it stops when the objective function acquires desired degree of accuracy. The performance of the proposed algorithm is illustrated with examples. The outcomes are compared with some existing models. The road map of the paper is as follows- section 2 describes the problem statement. Section 3, illustrates the preliminaries for the proposed technique. Section 4, proposes the algorithm. Section 5, 
describes the performance evaluation and comparisons with existing works and at last section 6 draws the conclusion.

\section{PROBLEM STATEMENT}

The problem addressed in the paper is concerned with allocation of tasks to processors of a distributed system with the goal of minimizing response time and system cost. The distributed system consists of multiple processors, where multiple users can work simultaneously from different sites. The processors available, at different sites in the system, process the requests according to availability. Each processor has its own computation capacity and memory while communication network has a limited communication capacity. In real time scenario, some failure rate is also associated with each processor and communication link. Figure 1 shows a general model of distributed system.

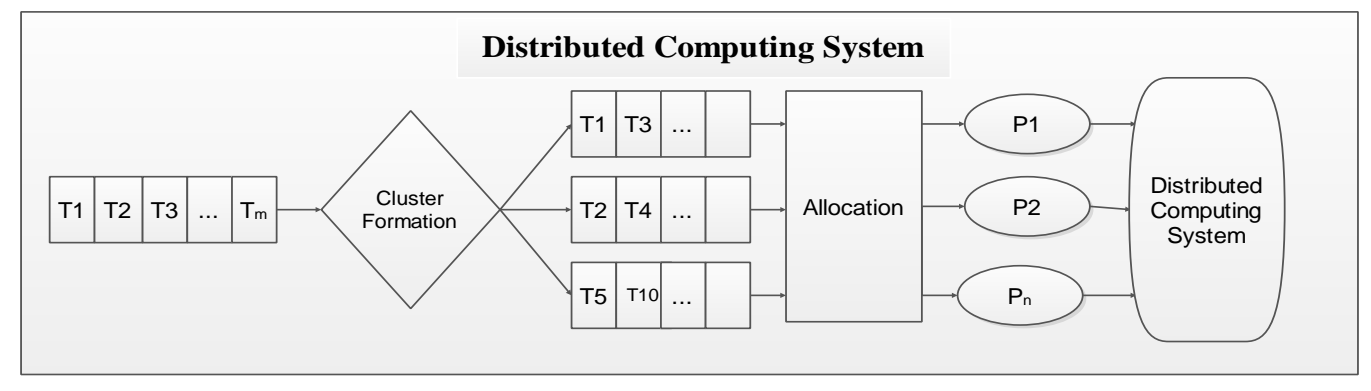

Figure 1. Distributed system model

Different factors are considered while allocating tasks to processors. Two main factors are Execution Time of tasks at different processors [7] and Inter Processor Communication (IPC) overhead [14, 15]. A set of $m$ tasks, to be executed parallel, are to be allocated to $n$ processors where $1 \leq i \leq m$, $1 \leq k \leq n \& m>n$. The tasks require processor resources such as computational capacity and memory capacity. The system resources have restricted capacity and a failure rate is associated with each component. The purpose of task allocation is to find optimal allocation of each task to the processors such that the system cost and response time are minimized with proper mapping of tasks to processors so that no processor remains idle. Furthermore, the task requirements and resource limitations are met.

\section{PRELIMINARIES}

\subsection{Execution Time (ET)}

The execution time, $e_{i k}$ is the amount of time taken by task $t_{i}$, which is to be executed on the processor $p_{k}$, where $1 \leq i \leq m$, $1 \leq k \leq n$. If a task $t_{i}$ is assigned to a processor $p_{k}$ but is not executed due to absence of some resources, then $e_{i k}$ of the task on the processor is taken to be $\infty$ i.e. very large value. The execution time, $e_{i k}$, of each task on each processor can be written in the form of Execution Time Matrix (ETM). The Total Execution Time (ET) is calculated as given [16]:

$$
E T=\sum_{i=1}^{m} \sum_{k=1}^{n} e_{i k} x_{i k}
$$

$x$ is an assignment matrix such that

$$
x_{i k}=\left\{\begin{array}{l}
1, \text { if task } T_{i} \text { is assigned to processor } p_{k} \\
0, \text { else }
\end{array}\right.
$$

\subsection{Inter Task Communication Time (ITCT)}

The Inter Task Communication Time, $c_{i j}$, is the amount of time incurred due to the data units exchanged between the tasks $t_{i}$ and $t_{j}$ if they are executed on different processors. When some tasks are assigned to same processor, then $c_{i j}=0$.
Total Inter-Task Communication Time (ITCT) of program is calculated by using Eq. (2) given as follows [16]:

$$
\operatorname{ITCT}=\sum_{i, j=1}^{m} \sum_{\substack{k, l=1 \\ k \neq l}}^{n} c_{i j} x_{i k} x_{j l}
$$

$x$ is an assignment matrix such that

$$
x_{i k}=\left\{\begin{array}{l}
1, \text { if task } t_{i} \text { is assigned to processor } p_{k} \\
0, \text { else }
\end{array}\right.
$$

\subsection{Response time (RT)}

Response time of a system is the amount of time taken by each processor for the computation of the given tasks including inter task communication time. It is defined by considering the processor with heaviest aggregate computation and communication loads of the processor. Response time (RT) of a system is calculated as follows:

$$
R T=\max \left\{\sum_{i=1}^{m} \sum_{k=1}^{n} e_{i k} x_{i k}+\sum_{i, j=1}^{m} \sum_{\substack{l=1 \\ k \neq l}}^{n} c_{i j} x_{i k} x_{j l}\right\}
$$

\subsection{System Cost (SC)}

The System Cost (SC) of the system is the sum of total execution time and total inter task communication time i.e.

$$
S C=\sum_{i=1}^{m} \sum_{k=1}^{n} e_{i k} x_{i k}+\sum_{i, j=1}^{m} \sum_{\substack{k, l=1 \\ k \neq l}}^{n} c_{i k} x_{i k} x_{j l}
$$

\subsection{Allocation constraints}

The allocation depends on tasks requirements and system resources. Some of the constraints are considered in the proposed algorithm and are as follows:

> Processor load constraints: For task assignment, the total processing load required by all tasks assigned to processor $k$ must be less than or equal to available computational load of processor $k$. If $L_{i}$ denotes the processing load required 
by task $i$ and if $p_{k}$ denotes available processing load of processor $k$, then the following inequality for each processor must hold:

$$
\sum_{i=1}^{m} L_{i} x_{i k} \leq P_{k}
$$

$x_{i k}$ is an assignment matrix.

> Number of clusters: To execute a program parallel in minimum time, all the processors must be utilized wisely and tasks should be allocated in such a way that no processor remains idle. Keeping this point in mind the maximum number of clusters, a system can have, should be equal to number of processors i.e. neither should it exceed the number of processor nor should it be less than that else some of the processors may remain idle.

$>$ Number of tasks in a processor: To execute a program parallel in minimum time and to balance load on all the processors, the maximum number of tasks in a cluster should be $\leq \frac{m}{n}$, where $m$ is the number of tasks and $n$ is the number of processors.

\section{PROPOSED WORK}

In this section, first the Fuzzy $C$-Means clustering technique have been discussed and then explains how it may be employed for task allocation.

\subsection{Fuzzy $C$-means clustering technique}

Clustering groups the objects of similar nature and the metric is supposed to be defined on nature of addressed problem. Clustering can be hierarchical or partitioned. Hierarchical clustering is organized as tree, having a set of nested clusters, while partitioned clustering is division of objects into non-overlapping cluster in such a way that each object is contained exactly in one cluster. But, sometimes to improve and optimize the solution, it becomes an essential requirement to shift an object/s from one cluster to some other cluster by taking into consideration the parameters, constraints and available resources. Thus, having a flexibility of an object, of being a member of other clusters too, makes the system more efficient. Fuzzy $C$-Means clustering provides this flexibility to the objects where data objects (points) are grouped into overlapping clusters. It is different from other techniques in a way that in this technique the data point can potentially belongs to multiple clusters with a variable degree of membership value in each cluster. So, if data points are located on the boundaries of the clusters, they are not forced to belong to a certain cluster and have flexibility of being the member of others clusters too, for better performance of system. Clusters are formed according to distance, between data points and cluster centers, which characterized by membership values of data points for different clusters. Larger distance of data point from cluster centre is characterized by smaller membership value and smaller distance of is characterized by larger membership value. Fuzzy $C$-Means (FCM) is an iterative process and it stops when the objective function acquires desired degree of accuracy.

This clustering is based on Zadeh's idea of fuzzy which was introduced on 1965. This algorithm does not classify fuzzy data, it classifies crisp data into fuzzy clusters. Fuzzy $C$-Means clustering technique can be summarized as below:

a) Generate $n$ clusters randomly b) Cluster centroids are calculated.

c) Finding Euclidean distance of each data point from each cluster centre.

d) Finding the membership value of each data point for each cluster, with the help of Euclidean distance.

e) Updating the clusters by taking membership value into consideration.

f) Computing new cluster centroid based on updated clusters.

g) Repeating the steps b) to f) until there is no change in the cluster centre or the difference of membership value is equal to the desired degree of accuracy.

\subsection{Proposed algorithm}

\subsubsection{Fetch the data set}

Fetch the data set. Inputs are:

$\begin{aligned} \text { i. } & \text { A program of } m \text { tasks i.e. } \mathrm{T}=\left\{t_{1}, t_{2}, t_{3}, \ldots, t_{m}\right\} . \\ \text { ii. } & \text { A set of } n \text { processors i.e. } \mathrm{P}=\left\{p_{1}, p_{2}, p_{3}, \ldots, p_{m}\right\} . \\ \text { iii. } & \text { A set of } n \text { clusters i.e. } \mathrm{G}=\left\{g_{1}, g_{2}, g_{3}, \ldots, g_{m}\right\} . \\ \text { iv. } & \mathrm{ET}\left(e_{i k}\right) \text { and } \mathrm{ITCT}\left(c_{i j}\right) \text { are taken in the form of } \\ & \text { matrices as Execution Time Matrix (ETM) and } \\ & \text { Inter Task Communication Time Matrix } \\ & \text { (ITCTM). }\end{aligned}$

\subsubsection{Fuzzy $C$-means clustering technique to form clusters}

Let $\mathrm{G}$ denotes the clusters and $\mathrm{T}$ denotes the tasks, then form a matrix $\mathrm{U}$ of order $G \times T$. Initializing Fuzzy $C$-Means (FCM) clustering technique by either forming the clusters randomly or using $K$-means clustering. In the clusters by Fuzzy $C$-means, the elements (i.e. tasks) belonging to one cluster may be shifted to another to balance the load and minimize the system cost, if required.

\subsubsection{Assignment of tasks using Hungarian method}

After forming clusters, the execution time (for each processor) and inter task communication time of each cluster is calculated. Then applying Hungarian method to allocate clusters to different processors in such a way that processor executes the clustered tasks in minimum time. If there is tie between two or more clustered tasks, the same above mentioned method can be used for allocation by using that combination which optimizes the system cost and response time.

\subsubsection{Determination of Process Response Time (PRT)}

The Process Response Time (PRT) is calculated using Eq. (6) as follows:

$$
\begin{aligned}
P R T_{k}=\min \left\{\left(E T_{i 1}\right.\right. & \left.+I T C T_{i 1}\right),\left(\left(E T_{i 2}\right.\right. \\
& \left.+I T C T_{i 2}\right), \ldots \ldots \ldots,\left(E T_{i m}\right. \\
& \left.\left.+I T C T_{i m}\right)\right\}
\end{aligned}
$$

Clustered Task $g_{i} \in G$ is assigned to that processor for which PRT, i.e. $\left(E T_{i k}+I T C T_{i j}\right)$, is minimum. This process is continued until all the clusters, $g_{k} \in G \forall 1 \leq k \leq n$ are assigned to all the processors.

\subsubsection{Determination of Overall Process Response Time (OPRT)} \& System Cost (SC)

When the procedure of assigning the clustered tasks to different processors gets over, the OPRT for the distribution is the maximum of Process Response Time i.e. 


$$
O P R T=\max \left\{P R T_{k}\right\} ; \forall 1 \leq k \leq n
$$

$$
S C=\sum_{k=1}^{n} \mathrm{PRT}_{k}
$$

The System Cost (SC) after assigning all clustered tasks is calculated using Eq. (8) as follows:
Flow Chart of the algorithm is shown in Figure 2.

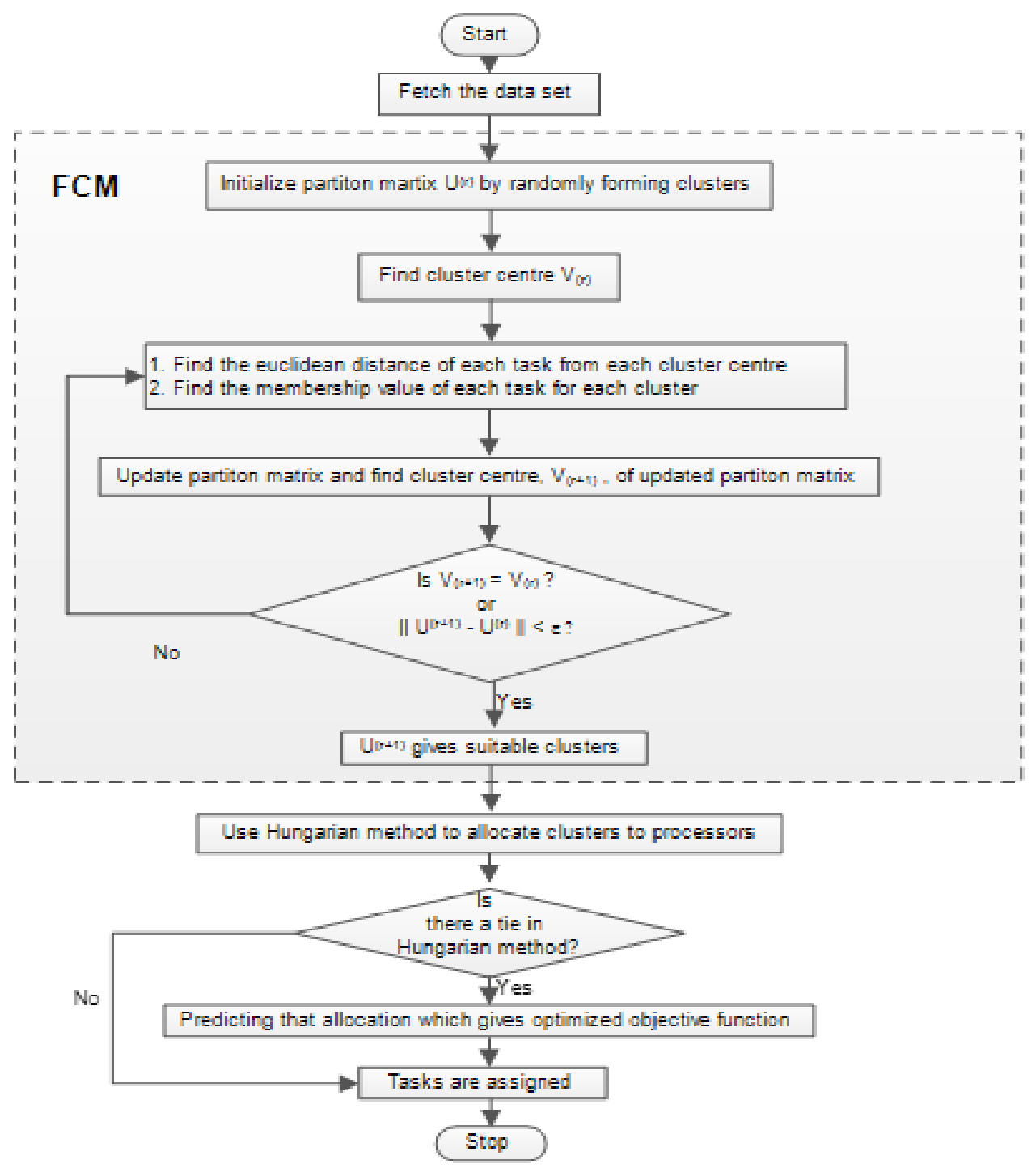

Figure 2. Flow chart of proposed algorithm

\section{PERFORMANCE ANALYSIS AND DISCUSSION}

This section illustrates the proposed algorithm with the help of examples.

Table 1. Execution time matrix

\begin{tabular}{|c|c|c|c|}
\hline $\begin{array}{c}\text { Processor } \rightarrow \\
\text { Tasks } \downarrow\end{array}$ & $p_{1}$ & $p_{2}$ & $p_{3}$ \\
\hline$t_{1}$ & 174 & 176 & 110 \\
\hline$t_{2}$ & 95 & 15 & 134 \\
\hline$t_{3}$ & 196 & 79 & 156 \\
\hline$t_{4}$ & 148 & 215 & 143 \\
\hline$t_{5}$ & 44 & 234 & 122 \\
\hline$t_{6}$ & 241 & 225 & 27 \\
\hline$t_{7}$ & 12 & 28 & 192 \\
\hline$t_{8}$ & 215 & 13 & 122 \\
\hline$t_{9}$ & 211 & 11 & 208 \\
\hline
\end{tabular}

Example 1: Consider a program made up of nine tasks $\left\{t_{1}, t_{2}, t_{3}, \ldots . t_{9}\right\}$ to be allocated to three processors $\left\{p_{1}, p_{2}, p_{3}\right\}$. The execution cost of each task on each processor and the inter - task communication cost between tasks is considered in the form of matrices as given in Table 1 above and Table 2 below.

Table 2. Inter - task communication time matrix

\begin{tabular}{|c|c|c|c|c|c|c|c|c|c|}
\hline $\begin{array}{c}\text { Tasks } \\
\rightarrow \\
\downarrow\end{array}$ & $t_{1}$ & $t_{2}$ & $t_{3}$ & $t_{4}$ & $t_{5}$ & $t_{6}$ & $t_{7}$ & $t_{8}$ & $t_{9}$ \\
\hline$t_{1}$ & 0 & 8 & 10 & 4 & 0 & 3 & 4 & 0 & 0 \\
\hline$t_{2}$ & 8 & 0 & 7 & 0 & 0 & 0 & 0 & 3 & 0 \\
\hline$t_{3}$ & 10 & 7 & 0 & 1 & 0 & 0 & 0 & 0 & 0 \\
\hline$t_{4}$ & 4 & 0 & 1 & 0 & 6 & 0 & 0 & 8 & 0 \\
\hline$t_{5}$ & 0 & 0 & 0 & 6 & 0 & 0 & 0 & 12 & 0 \\
\hline$t_{6}$ & 3 & 0 & 0 & 0 & 0 & 0 & 0 & 0 & 12 \\
\hline$t_{7}$ & 4 & 0 & 0 & 0 & 0 & 0 & 0 & 3 & 10 \\
\hline$t_{8}$ & 0 & 3 & 0 & 8 & 12 & 0 & 3 & 0 & 5 \\
\hline$t_{9}$ & 0 & 0 & 0 & 0 & 0 & 12 & 10 & 5 & 0 \\
\hline
\end{tabular}


While using Fuzzy C - Means clustering technique, the partition matrix at each iteration (showing membership values of each task in each cluster) and the matrix of cluster centres are shown in Table 3 and Table 4.

Table 3. Iterations of partition matrix (showing membership values)

\begin{tabular}{|c|c|c|c|c|c|c|c|c|c|c|}
\hline \multirow{3}{*}{ U1 } & $g_{1}$ & 0.26944 & 0.2953 & 0.69726 & 0.11647 & 0.2157 & 0.24482 & 0.34089 & 0.40289 & 0.25013 \\
\hline & $g_{2}$ & 0.65614 & 0.05707 & 0.05908 & 0.83766 & 0.6651 & 0.6326 & 0.14511 & 0.07719 & 0.05999 \\
\hline & $g_{3}$ & 0.07442 & 0.64763 & 0.24366 & 0.04587 & 0.1192 & 0.12258 & 0.514 & 0.51992 & 0.68988 \\
\hline & & & & & & & & & & \\
\hline \multirow{3}{*}{ U2 } & $g_{1}$ & 0.13282 & 0.25301 & 0.82182 & 0.05699 & 0.19409 & 0.23627 & 0.32528 & 0.46318 & 0.35981 \\
\hline & $g_{2}$ & 0.81396 & 0.05594 & 0.03873 & 0.91363 & 0.66779 & 0.62349 & 0.15925 & 0.07537 & 0.07084 \\
\hline & $g_{3}$ & 0.05322 & 0.69105 & 0.13945 & 0.02938 & 0.13812 & 0.14024 & 0.51547 & 0.46145 & 0.56935 \\
\hline \multirow{3}{*}{ U3 } & $g_{1}$ & 0.09418 & 0.1558 & 0.91397 & 0.04361 & 0.17362 & 0.22585 & 0.27523 & 0.59509 & 0.51028 \\
\hline & $g_{2}$ & 0.85947 & 0.03807 & 0.02343 & 0.92942 & 0.67249 & 0.63271 & 0.14793 & 0.07202 & 0.075 \\
\hline & $g_{3}$ & 0.04635 & 0.80613 & 0.0626 & 0.02697 & 0.15389 & 0.14144 & 0.57684 & 0.33289 & 0.41472 \\
\hline \multirow{3}{*}{ U4 } & $g_{1}$ & 0.07885 & 0.04934 & 0.94406 & 0.03894 & 0.1554 & 0.21915 & 0.18803 & 0.74374 & 0.67206 \\
\hline & $g_{2}$ & 0.87947 & 0.01416 & 0.02079 & 0.93429 & 0.67186 & 0.64786 & 0.11284 & 0.06565 & 0.0748 \\
\hline & $g_{3}$ & 0.04168 & 0.9365 & 0.03515 & 0.02677 & 0.17274 & 0.13299 & 0.69913 & 0.19061 & 0.25314 \\
\hline \multirow{3}{*}{ U5 } & $g_{1}$ & 0.06728 & 0.06102 & 0.91672 & 0.0361 & 0.14294 & 0.20859 & 0.12142 & 0.83341 & 0.77084 \\
\hline & $g_{2}$ & 0.8959 & 0.01931 & 0.03803 & 0.9374 & 0.67022 & 0.66874 & 0.07791 & 0.05469 & 0.06697 \\
\hline & $g_{3}$ & 0.03682 & 0.91967 & 0.04525 & 0.0265 & 0.18684 & 0.12267 & 0.80067 & 0.1119 & 0.16219 \\
\hline \multirow{4}{*}{ U6 } & & & & & & & & & & \\
\hline & $g_{1}$ & 0.05997 & 0.10523 & 0.88754 & 0.03562 & 0.13794 & 0.19827 & 0.08842 & 0.86924 & 0.8124 \\
\hline & $g_{2}$ & 0.90628 & 0.03409 & 0.05572 & 0.93707 & 0.6669 & 0.6861 & 0.05756 & 0.04801 & 0.06017 \\
\hline & $g_{3}$ & 0.03375 & 0.86068 & 0.05674 & 0.02731 & 0.19516 & 0.11563 & 0.85402 & 0.08275 & 0.12743 \\
\hline \multirow{3}{*}{ U7 } & $g_{1}$ & 0.05587 & 0.13943 & 0.87322 & 0.03631 & 0.13676 & 0.19187 & 0.07063 & 0.88326 & 0.83039 \\
\hline & $g_{2}$ & 0.9125 & 0.04515 & 0.06542 & 0.93536 & 0.66271 & 0.69707 & 0.04585 & 0.0455 & 0.05691 \\
\hline & $g_{3}$ & 0.03163 & 0.81542 & 0.06136 & 0.02833 & 0.20053 & 0.11106 & 0.88352 & 0.07124 & 0.1127 \\
\hline & & & & & & & & & & \\
\hline \multirow{3}{*}{ U8 } & $g_{1}$ & 0.05353 & 0.16252 & 0.86763 & 0.03715 & 0.13692 & 0.18832 & 0.06047 & 0.88926 & 0.8387 \\
\hline & $g_{2}$ & 0.9163 & 0.05237 & 0.06996 & 0.93375 & 0.65916 & 0.70357 & 0.03904 & 0.0447 & 0.05559 \\
\hline & $g_{3}$ & 0.03017 & 0.78511 & 0.06241 & 0.0291 & 0.20392 & 0.10811 & 0.90049 & 0.06604 & 0.10571 \\
\hline & & & & & & & & & & \\
\hline \multirow{3}{*}{ U9 } & $g_{1}$ & 0.0522 & 0.17753 & 0.86573 & 0.03783 & 0.13737 & 0.18637 & 0.0545 & 0.89213 & 0.84274 \\
\hline & $g_{2}$ & 0.9186 & 0.05692 & 0.07202 & 0.93258 & 0.65665 & 0.70738 & 0.03501 & 0.04448 & 0.05511 \\
\hline & $g_{3}$ & 0.0292 & 0.76555 & 0.06225 & 0.02959 & 0.20598 & 0.10625 & 0.91049 & 0.06339 & 0.10215 \\
\hline \multirow{3}{*}{ U10 } & $g_{1}$ & 0.05144 & 0.18711 & 0.86516 & 0.0383 & 0.13778 & 0.18529 & 0.05091 & 0.89362 & 0.84479 \\
\hline & $g_{2}$ & 0.91997 & 0.05975 & 0.07298 & 0.93181 & 0.65501 & 0.70963 & 0.03259 & 0.04444 & 0.05495 \\
\hline & $g_{3}$ & 0.02859 & 0.75314 & 0.06186 & 0.02989 & 0.20721 & 0.10508 & 0.9165 & 0.06194 & 0.10026 \\
\hline
\end{tabular}

Table 4. Iterations of cluster centres

\begin{tabular}{|c|c|c|c|c|c|c|c|c|c|}
\hline \multirow{2}{*}{$\begin{array}{c}\text { Iterations } \\
\downarrow\end{array}$} & \multirow{2}{*}{$\begin{array}{c}\text { No. of } \\
\text { Clusters }\end{array}$} & \multicolumn{3}{|c|}{ Coordinates } & \multirow{2}{*}{$\begin{array}{c}\text { Iterations } \\
\downarrow\end{array}$} & \multirow{2}{*}{$\begin{array}{c}\text { No. of } \\
\text { Clusters }\end{array}$} & \multicolumn{3}{|c|}{ Coordinates } \\
\hline & & $x$ & $y$ & $Z$ & & & $x$ & $y$ & $z$ \\
\hline \multirow{3}{*}{ Center 1} & $g_{1}$ & 155 & 90 & 133.33333 & \multirow{3}{*}{ Center 6} & $g_{1}$ & 203.98745 & 44.22856 & 156.54067 \\
\hline & $g_{2}$ & 144.33333 & 224.66667 & 97.33333 & & $g_{2}$ & 153.95814 & 206.81627 & 109.35136 \\
\hline & $g_{3}$ & 146 & 17.33333 & 174 & & $g_{3}$ & 64.68694 & 27.34281 & 157.40131 \\
\hline \multirow{3}{*}{ Center 2} & $g_{1}$ & 166.38193 & 77.53152 & 144.41094 & \multirow{3}{*}{ Center 7} & $g_{1}$ & 204.94189 & 41.08747 & 157.21112 \\
\hline & $g_{2}$ & 148.08071 & 209.34167 & 108.82608 & & $g_{2}$ & 155.18978 & 206.87612 & 108.50419 \\
\hline & $g_{3}$ & 143.31387 & 23.10518 & 164.60876 & & $g_{3}$ & 57.98387 & 28.60875 & 160.8326 \\
\hline \multirow{3}{*}{ Center 3} & $g_{1}$ & 177.83962 & 66.48551 & 150.24886 & \multirow{3}{*}{ Center 8} & $g_{1}$ & 205.05052 & 39.66569 & 157.5271 \\
\hline & $g_{2}$ & 149.75095 & 206.05552 & 111.43946 & & $g_{2}$ & 156.05533 & 206.78809 & 107.9489 \\
\hline & $g_{3}$ & 128.69962 & 23.87541 & 159.98489 & & $g_{3}$ & 53.94841 & 29.49065 & 163.19529 \\
\hline \multirow{3}{*}{ Center 4} & $g_{1}$ & 190.9272 & 58.74352 & 153.35347 & \multirow{3}{*}{ Center 9} & $g_{1}$ & 204.92854 & 39.02938 & 157.66113 \\
\hline & $g_{2}$ & 150.84279 & 205.81643 & 111.27492 & & $g_{2}$ & 156.62046 & 206.68726 & 107.60592 \\
\hline & $g_{3}$ & 101.04029 & 25.29404 & 155.51662 & & $g_{3}$ & 51.45863 & 30.05883 & 164.71614 \\
\hline \multirow{3}{*}{ Center 5} & $g_{1}$ & 200.37714 & 50.38906 & 155.29311 & \multirow{3}{*}{ Center 10} & $g_{1}$ & 204.77892 & 38.73451 & 157.71129 \\
\hline & $g_{2}$ & 152.37622 & 206.38704 & 110.41493 & & $g_{2}$ & 156.97301 & 206.61035 & 107.39854 \\
\hline & $g_{3}$ & 76.93313 & 26.05557 & 154.03158 & & $g_{3}$ & 49.91486 & 30.4147 & 165.67657 \\
\hline
\end{tabular}

Since the convergence criterion $\left\|U^{(r+1)}-U^{(r)}\right\|<0.01$ fulfills at the tenth iteration and also cluster centres at two successive iterations, i.e. $9^{\text {th }}$ and $10^{\text {th }}$, are approximate same, therefore the procedure stops at $10^{\text {th }}$ step. The cluster formed, on the basis of membership values, are given in Table 5 below: 
Table 5. Formation of clusters

\begin{tabular}{|c|c|}
\hline Clusters & Tasks \\
\hline$g_{1}$ & $t_{3}+t_{8}+t_{9}$ \\
\hline$g_{2}$ & $t_{1}+t_{4}+t_{6}$ \\
\hline$g_{3}$ & $t_{2}+t_{5}+t_{7}$ \\
\hline
\end{tabular}

To allocate the clustered tasks to processors, Hungarian method is used. The Execution Time Matrix for clustered tasks and final allocation is shown in Table 6 given above.

Final allocation is: $g_{1} \rightarrow p_{2} ; g_{2} \rightarrow p_{3} ; g_{3} \rightarrow p_{1}$.

The final allocation task list for overall process response time and system cost is given in Table 7.
Table 6. Allocation matrix using Hungarian method

\begin{tabular}{|c|c|c|c|}
\hline Clusters & $\boldsymbol{p}_{\mathbf{1}}$ & $\boldsymbol{p}_{\mathbf{2}}$ & $\boldsymbol{p}_{\mathbf{3}}$ \\
\hline$g_{1}\left(t_{3}+t_{8}+t_{9}\right)$ & 622 & $\mathbf{1 0 3}$ & 486 \\
\hline$g_{2}\left(t_{1}+t_{4}+t_{6}\right)$ & 563 & 616 & $\mathbf{2 8 0}$ \\
\hline$g_{3}\left(t_{2}+t_{5}+t_{7}\right)$ & $\mathbf{1 5 1}$ & 277 & 448 \\
\hline
\end{tabular}

Example 2: Consider a program made up of ten tasks $\left\{t_{1}, t_{2}, t_{3}, \ldots, t_{10}\right\}$ to be allocated to three processors $\left\{p_{1}, p_{2}, p_{3}\right\}$. The execution cost of each task on each processor and the inter - task communication cost between tasks is considered in the form of matrices as shown in Table 8 and Table 9.

Table 7. Final task allocation with OPRT \& SC

\begin{tabular}{|c|c|c|c|c|c|c|}
\hline Processors & Clustered Tasks & $\begin{array}{c}\text { ET } \\
(\mathbf{1})\end{array}$ & $\begin{array}{c}\text { ITCT } \\
(\mathbf{2})\end{array}$ & $\begin{array}{c}\text { PRT=ET+ ITCT } \\
(\mathbf{1})+(\mathbf{2})\end{array}$ & OPRT & System Cost \\
\cline { 1 - 5 }$p_{1}$ & $\begin{array}{c}g_{3} \\
\left(t_{2}+t_{5}+t_{7}\right)\end{array}$ & 151 & 53 & 204 & \multirow{2}{*}{329} & \multirow{2}{*}{702} \\
\cline { 1 - 5 }$p_{2}$ & $\begin{array}{c}g_{1} \\
\left(t_{3}+t_{8}+t_{9}\right)\end{array}$ & 103 & 66 & 169 & \\
\cline { 1 - 4 }$p_{3}$ & $\begin{array}{c}g_{2} \\
\left(t_{1}+t_{4}+t_{6}\right)\end{array}$ & 280 & 49 & $\mathbf{3 2 9}$ & & \\
\hline
\end{tabular}

Table 8. Execution time matrix

\begin{tabular}{|c|c|c|c|}
\hline $\begin{array}{c}\text { Processor } \rightarrow \\
\text { Tasks } \downarrow\end{array}$ & $p_{1}$ & $p_{2}$ & $p_{3}$ \\
\hline$t_{1}$ & 14 & 16 & 9 \\
\hline$t_{2}$ & 13 & 19 & 18 \\
\hline$t_{3}$ & 11 & 13 & 19 \\
\hline$t_{4}$ & 13 & 8 & 17 \\
\hline$t_{5}$ & 12 & 13 & 10 \\
\hline$t_{6}$ & 13 & 16 & 9 \\
\hline$t_{7}$ & 7 & 15 & 11 \\
\hline$t_{8}$ & 5 & 11 & 14 \\
\hline$t_{9}$ & 18 & 12 & 20 \\
\hline$t_{10}$ & 21 & 7 & 16 \\
\hline
\end{tabular}

Table 9. Inter - task communication matrix

\begin{tabular}{|c|c|c|c|c|c|c|c|c|c|c|}
\hline $\begin{array}{c}\text { Tasks } \\
\downarrow\end{array}$ & $t_{1}$ & $t_{2}$ & $t_{3}$ & $t_{4}$ & $t_{5}$ & $t_{6}$ & $t_{7}$ & $t_{8}$ & $t_{9}$ & $t_{10}$ \\
\hline$t_{1}$ & 0 & 18 & 12 & 9 & 11 & 14 & 0 & 0 & 0 & 0 \\
\hline$t_{2}$ & 18 & 0 & 0 & 0 & 0 & 0 & 0 & 19 & 16 & 0 \\
\hline$t_{3}$ & 12 & 0 & 0 & 0 & 0 & 0 & 23 & 0 & 0 & 0 \\
\hline$t_{4}$ & 9 & 0 & 0 & 0 & 0 & 0 & 0 & 27 & 23 & 0 \\
\hline$t_{5}$ & 11 & 0 & 0 & 0 & 0 & 0 & 0 & 0 & 13 & 0 \\
\hline$t_{6}$ & 14 & 0 & 0 & 0 & 0 & 0 & 0 & 15 & 0 & 0 \\
\hline$t_{7}$ & 0 & 0 & 23 & 0 & 0 & 0 & 0 & 0 & 0 & 17 \\
\hline$t_{8}$ & 0 & 19 & 0 & 27 & 0 & 15 & 0 & 0 & 0 & 11 \\
\hline$t_{9}$ & 0 & 16 & 0 & 23 & 13 & 0 & 0 & 0 & 0 & 13 \\
\hline$t_{10}$ & 0 & 0 & 0 & 0 & 0 & 0 & 17 & 11 & 13 & 0 \\
\hline
\end{tabular}

Table 10. Iterations of partition matrix (showing membership values)

\begin{tabular}{|c|c|r|r|r|r|r|r|r|r|r|r|}
\hline \multirow{3}{*}{ U1 } & $g_{1}$ & 0.84017 & 0.5733 & 0.50007 & 0.13976 & 0.06266 & 0.19189 & 0.25357 & 0.24949 & 0.38848 & 0.24435 \\
\cline { 2 - 12 } & $g_{2}$ & 0.0596 & 0.23876 & 0.13938 & 0.2045 & 0.81031 & 0.59174 & 0.46161 & 0.36673 & 0.21639 & 0.32659 \\
\cline { 2 - 12 } & $g_{3}$ & 0.10023 & 0.18794 & 0.36055 & 0.65574 & 0.12703 & 0.21637 & 0.28482 & 0.38378 & 0.39513 & 0.42906 \\
\hline \multirow{3}{*}{ U2 } & $g_{1}$ & 0.855 & 0.50679 & 0.52748 & 0.1051 & 0.03285 & 0.14646 & 0.20051 & 0.25203 & 0.42655 & 0.27588 \\
\cline { 2 - 12 } & $g_{2}$ & 0.05209 & 0.32737 & 0.11666 & 0.08618 & 0.92586 & 0.73209 & 0.61762 & 0.41518 & 0.14569 & 0.2375 \\
\cline { 2 - 11 } & $g_{3}$ & 0.09291 & 0.16584 & 0.35586 & 0.80872 & 0.04129 & 0.12145 & 0.18187 & 0.33279 & 0.42776 & 0.48662 \\
\hline U3 & $g_{1}$ & 0.89767 & 0.4694 & 0.61314 & 0.06736 & 0.03558 & 0.1099 & 0.15155 & 0.25851 & 0.43318 & 0.26059 \\
\hline
\end{tabular}




\begin{tabular}{|c|c|c|c|c|c|c|c|c|c|c|c|}
\hline & & 03751 & 0.38243 & 0.1053 & 0.04074 & 92818 & 0.80949 & 73197 & 0.45736 & & \\
\hline & $g_{2}$ & 06482 & 0.14817 & 0.28156 & & 03624 & 0.08061 & 11648 & 0.28413 & 46113 & 0.56042 \\
\hline \multirow{3}{*}{ U4 } & $g_{1}$ & & & & & & & & & & \\
\hline & & 03209 & 0.41138 & & & .90433 & & & & & \\
\hline & $g_{3}$ & & & & & & & & & & \\
\hline \multirow{3}{*}{ U5 } & $g_{l}$ & & 0.44287 & 74 & & & & & & & \\
\hline & & & & & & & & & & & \\
\hline & $g_{3}$ & & & & & & & & & & \\
\hline \multirow{3}{*}{ U6 } & $g_{1}$ & & & & 0.15 & & & & & & \\
\hline & $g_{2}$ & & & & & & & & & & \\
\hline & $g_{3}$ & & & & & & & & & & \\
\hline \multirow{3}{*}{ U7 } & $g_{l}$ & & & & & & & & & & \\
\hline & $g_{2}$ & & & & & & & & & & 948 \\
\hline & & & & & & & & & & & \\
\hline \multirow{3}{*}{ U8 } & $g_{1}$ & & & & & & & & & & \\
\hline & & & & & & & & & & & \\
\hline & $g_{3}$ & & & & & & & & & & \\
\hline \multirow{3}{*}{ U9 } & $g_{1}$ & & & & & & & & & & \\
\hline & s. & & & & & & & & & & \\
\hline & $g_{3}$ & & & & & & & & & & 333 \\
\hline \multirow{3}{*}{ U10 } & $g_{1}$ & & & & & & & & & & \\
\hline & $g_{2}$ & & & & & & & & & & \\
\hline & $g_{3}$ & & & & & & & & & & \\
\hline \multirow{3}{*}{ U11 } & $g_{1}$ & & & & & & & & & & \\
\hline & & & & & & & & & & & \\
\hline & $g_{3}$ & & & & & & & & & & \\
\hline \multirow{3}{*}{ U12 } & $g_{1}$ & 0.85467 & & & & & & & & & 3099 \\
\hline & & & & & & & & & & & \\
\hline & $g_{3}$ & & & & & & & & & & \\
\hline \multirow{3}{*}{ U13 } & $g_{1}$ & & & & & & & & & & \\
\hline & $g_{2}$ & & & & & & & & & & \\
\hline & & & & & & & & & & & \\
\hline \multirow{3}{*}{ U14 } & & & & & & & & & & & \\
\hline & & & & & & & & & & & \\
\hline & & & & 0.04725 & 0.46403 & 0.04658 & 0.07311 & 0.0436 & 0.1331 & 0.64267 & \\
\hline
\end{tabular}

Table 11. Iterations of cluster centres

\begin{tabular}{|c|c|c|c|c|}
\hline Iterations & & No. of & \multicolumn{3}{|c|}{ Coordinates } \\
\cline { 2 - 5 } & Clusters & $x$ & $y$ & $z$ \\
\hline \multirow{4}{*}{ Center 1 } & $g_{1}$ & 12.66667 & 16 & 17.33333 \\
\cline { 2 - 5 } & $g_{2}$ & 12.66667 & 12.33333 & 12 \\
\cline { 2 - 5 } & $g_{3}$ & 12.75 & 11.25 & 15.25 \\
\hline \multirow{4}{*}{ Center 2 } & $g_{1}$ & 13.32716 & 15.13869 & 17.25228 \\
\cline { 2 - 5 } & $g_{2}$ & 11.79793 & 13.40774 & 11.37251 \\
\cline { 2 - 5 } Center 3 & $g_{3}$ & 13.26053 & 10.44493 & 16.22578 \\
\cline { 2 - 5 } & $g_{1}$ & 13.5674 & 14.84055 & 17.66616 \\
\cline { 2 - 5 } & $g_{2}$ & 11.14801 & 14.05792 & 10.79482 \\
\cline { 2 - 5 } Center 4 & $g_{3}$ & 14.05844 & 9.56795 & 16.88512 \\
\cline { 2 - 5 } & $g_{1}$ & 13.48814 & 14.73917 & 17.99345 \\
\cline { 2 - 5 } & $g_{2}$ & 10.78603 & 14.33411 & 10.70182 \\
\hline \multirow{3}{*}{ Center 5 } & $g_{3}$ & 14.76034 & 9.04835 & 17.02626 \\
\cline { 2 - 5 } & $g_{1}$ & 13.2945 & 14.68999 & 18.13227 \\
\cline { 2 - 5 } Center 6 & $g_{2}$ & 10.57464 & 14.42635 & 10.74227 \\
\hline & $g_{3}$ & 15.3709 & 8.84049 & 17.03303 \\
\cline { 2 - 5 } & $g_{1}$ & 13.09276 & 14.66078 & 18.16463 \\
\cline { 2 - 5 } & $g_{2}$ & 10.43981 & 14.44923 & 10.78314 \\
\hline \multirow{3}{*}{ Center 7 } & $g_{3}$ & 15.95703 & 8.78626 & 17.0564 \\
\cline { 2 - 5 } & $g_{1}$ & 12.90375 & 14.63101 & 18.15025 \\
\cline { 2 - 5 } & $g_{2}$ & 10.34777 & 14.44649 & 10.81186 \\
\hline \multirow{3}{*}{ Center 8 } & $g_{3}$ & 16.53335 & 8.79949 & 17.10356 \\
\cline { 2 - 5 } & $g_{1}$ & 12.73741 & 14.58346 & 18.11863 \\
\cline { 2 - 5 } & $g_{2}$ & 10.28437 & 14.43494 & 10.83394 \\
\hline \multirow{3}{*}{ Center 9 } & $g_{3}$ & 17.07674 & 8.83754 & 17.16028 \\
\cline { 2 - 5 } & $g_{1}$ & 12.60214 & 14.51304 & 18.08541 \\
\hline & $g_{2}$ & 10.24407 & 14.42292 & 10.85312 \\
\hline
\end{tabular}

\begin{tabular}{|l|l|c|c|c|}
\hline & $g_{3}$ & 17.54509 & 8.87491 & 17.21059 \\
\hline \multirow{3}{*}{ Center 10 } & $g_{1}$ & 12.5012 & 14.42774 & 18.05933 \\
\cline { 2 - 5 } & $g_{2}$ & 10.22334 & 14.41557 & 10.86976 \\
\cline { 2 - 5 } & $g_{3}$ & 17.90701 & 8.8971 & 17.2431 \\
\hline \multirow{3}{*}{ Center 11 } & $g_{1}$ & 12.43084 & 14.34138 & 18.04338 \\
\cline { 2 - 5 } & $g_{2}$ & 10.21783 & 14.41503 & 10.88282 \\
\cline { 2 - 5 } Center 12 & $g_{3}$ & 18.16176 & 8.90151 & 17.25597 \\
\hline & $g_{1}$ & 12.38353 & 14.26466 & 18.03627 \\
\cline { 2 - 5 } & $g_{2}$ & 10.22241 & 14.42039 & 10.89183 \\
\cline { 2 - 5 } Center 13 & $g_{3}$ & 18.33143 & 8.89318 & 17.25458 \\
\cline { 2 - 5 } & $g_{1}$ & 12.35188 & 14.20202 & 18.03491 \\
\cline { 2 - 5 } Center 14 & $g_{2}$ & 10.23242 & 14.42907 & 10.89734 \\
\hline & $g_{3}$ & 18.44266 & 8.87884 & 17.24592 \\
\cline { 2 - 5 } & $g_{1}$ & 12.33037 & 14.15323 & 18.03641 \\
\cline { 2 - 5 } & $g_{2}$ & 10.24443 & 14.43871 & 10.90034 \\
\hline & $g_{3}$ & 18.5163 & 8.86334 & 17.23507 \\
\hline
\end{tabular}

While using Fuzzy $C$-Means clustering technique, the partition matrix at each iteration (showing membership values of each task in each cluster) and the matrix of cluster centres are shown in Table 10 and Table 11.

Table 12. Formation of clusters

\begin{tabular}{|c|c|}
\hline Clusters & Tasks \\
\hline$g_{1}$ & $t_{2}+t_{3}+t_{7}$ \\
\hline$g_{2}$ & $t_{4}+t_{8}+t_{9}+t_{10}$ \\
\hline$g_{3}$ & $t_{1}+t_{5}+t_{6}$ \\
\hline
\end{tabular}


Since the convergence criterion $\left\|U^{(r+1)}-U^{(r)}\right\|<0.01$ fulfills at the fourteenth iteration and also cluster centres at two successive iterations, i.e. $13^{\text {th }}$ and $14^{\text {th }}$, are approximate same, therefore the procedure stops at $14^{\text {th }}$ step. The cluster formed, on the basis of membership values, are given in Table 12 .

To allocate the clustered tasks to processors, Hungarian method is used. The Execution Time Matrix for clustered tasks and final allocation is shown in Table 13.

Table 13. Allocation matrix using Hungarian matrix

\begin{tabular}{|c|c|c|c|}
\hline Clusters & $\boldsymbol{p}_{\mathbf{1}}$ & $\boldsymbol{p}_{\mathbf{2}}$ & $\boldsymbol{p}_{\mathbf{3}}$ \\
\hline$g_{1}\left(t_{2}+t_{3}+t_{7}\right)$ & $\mathbf{3 1}$ & 47 & 48 \\
\hline$g_{2}\left(t_{4}+t_{8}+t_{9}+t_{10}\right)$ & 57 & $\mathbf{3 8}$ & 67 \\
\hline$g_{3}\left(t_{1}+t_{5}+t_{6}\right)$ & 39 & 51 & $\mathbf{2 8}$ \\
\hline
\end{tabular}

Final allocation is: $g_{1} \rightarrow p_{1} ; g_{2} \rightarrow p_{2} ; g_{3} \rightarrow p_{3}$.

The final allocation task list for overall process response time and system cost is given in Table 14 below.

Task scheduling in a distributed system is challenging. Since there are more than one processor and large number of tasks are to be allocated. Keeping in mind the various restriction and conditions, it is difficult to meet all the objectives simultaneously. A lot of studies have been done for task scheduling in distributed system so that the response time and system cost can be reduced, load can be balanced, system reliability can be improved. Kumar et al. [16] proposed a technique to achieve optimal cost and optimal system reliability. The computational analysis is done to achieve the objective. Sriramdas et al. [5] proposed a model for reliability allocation technique using fuzzy model and an approximation method based on linear programming approach. The model is based on centralized distributed system (DS). Srinivasan and Geetharamani [17] proposed a technique to optimize the system cost of a fuzzy assignment problem which is formulated to crisp assignment problem in the form of linear programming problem (LPP) and then solving the problem using Robust Ranking method and Ones Assignment method. The results are illustrated with numerical examples. Qinma et al. [18] proposed an iterative greedy algorithm to maximize the system reliability by considering the wide range of parameters. The model has been simulated using MATLAB. Rehman et al. [19] proposed Min-Min algorithm for efficient resource distribution and load balancing. The results are then simulated and compared with Round Robin algorithm. Jang et al. [20] proposes a task scheduling model based on the genetic algorithm for an optimal task scheduling. The experimental results are then compared with existing task scheduling models. The proposed study presents an algorithm based on clustering technique. The proposed algorithm improves an overall process response time and system cost for unsupervised data by allocating the clustered tasks on processors with on an average balanced load. For this purpose, Execution Time and Inter Task Communication Time have been taken into consideration. The algorithm uses fuzzy $\mathrm{C}$ - means clustering technique for grouping the tasks. Later, to allocate clusters to processors, Hungarian method is used. From the data sets given in illustrated examples it can be seen that this algorithm improves the total response time and system cost. The proposed model is compared with the existing model, taken from research paper. Results are summarized as given in Table 15 .

The comparison of response time \& system cost is graphically shown in Figure 3 6.

Table 14. Final task allocation with OPRT \& SC

\begin{tabular}{|c|c|c|c|c|c|c|}
\hline Processors & Clustered Tasks & $\begin{array}{c}\text { ET } \\
(\mathbf{1})\end{array}$ & $\begin{array}{c}\text { ITCT } \\
(\mathbf{2})\end{array}$ & $\begin{array}{c}\text { PRT=ET+ ITCT } \\
(\mathbf{1})+(\mathbf{2})\end{array}$ & OPRT & System Cost \\
\cline { 1 - 5 }$p_{1}$ & $\begin{array}{c}g_{1} \\
\left(t_{1}+t_{2}+t_{3}+t_{8}\right)\end{array}$ & 31 & 82 & 113 & \multirow{2}{*}{127} & \multirow{2}{*}{335} \\
\cline { 1 - 5 }$p_{2}$ & $\begin{array}{c}g_{3} \\
\left(t_{4}+t_{9}+t_{10}\right)\end{array}$ & 38 & 89 & $\mathbf{1 2 7}$ & \\
\cline { 1 - 4 }$p_{3}$ & $\begin{array}{c}g_{2} \\
\left(t_{5}+t_{6}+t_{7}\right)\end{array}$ & 28 & 67 & 95 & \\
\hline
\end{tabular}

Table 15. Comparative study

\begin{tabular}{|c|c|c|c|c|c|}
\hline S.No. & Example & Processor & Tasks & Response Time & System Cost \\
\hline \multirow{9}{*}{1.} & \multirow{3}{*}{ Elsadek Model (1999) } & $p_{1}$ & $t_{6}+t_{7}+t_{9}$ & \multirow{3}{*}{479} & \multirow{3}{*}{1369} \\
\hline & & $p_{2}$ & $t_{4}+t_{5}+t_{8}$ & & \\
\hline & & $p_{3}$ & $t_{1}+t_{2}+t_{3}$ & & \\
\hline & \multirow{3}{*}{ H. Kumar Model (2018) } & $p_{1}$ & $t_{4}+t_{5}+t_{8}$ & \multirow{3}{*}{423} & \multirow{3}{*}{1109} \\
\hline & & $p_{2}$ & $t_{6}+t_{7}+t_{9}$ & & \\
\hline & & $p_{3}$ & $t_{1}+t_{2}+t_{3}$ & & \\
\hline & \multirow{3}{*}{ Proposed Algorithm } & $p_{1}$ & $t_{2}+t_{5}+t_{7}$ & \multirow{3}{*}{329} & \multirow{3}{*}{702} \\
\hline & & $p_{2}$ & $t_{3}+t_{8}+t_{9}$ & & \\
\hline & & $p_{3}$ & $t_{1}+t_{4}+t_{6}$ & & \\
\hline \multirow{9}{*}{2.} & \multirow{3}{*}{ Topcuoglu et. al. (2002) } & $p_{1}$ & $t_{5}+t_{7}$ & \multirow{3}{*}{172} & \multirow{3}{*}{335} \\
\hline & & $p_{2}$ & $t_{1}+t_{2}+t_{5}+t_{9}+t_{10}$ & & \\
\hline & & $p_{3}$ & $t_{4}+t_{6}+t_{8}$ & & \\
\hline & \multirow{3}{*}{ H. Kumar Model (2018) } & $p_{1}$ & $t_{3}+t_{7}+t_{10}$ & \multirow{3}{*}{130} & \multirow{3}{*}{332} \\
\hline & & $p_{2}$ & $t_{4}+t_{8}+t_{9}$ & & \\
\hline & & $p_{3}$ & $t_{1}+t_{2}+t_{5}+t_{6}$ & & \\
\hline & \multirow{3}{*}{ Proposed Algorithm } & $p_{1}$ & $t_{2}+t_{3}+t_{7}$ & \multirow{3}{*}{127} & \multirow{3}{*}{335} \\
\hline & & $p_{2}$ & $t_{4}+t_{8}+t_{9}+t_{10}$ & & \\
\hline & & $p_{3}$ & $t_{1}+t_{5}+t_{6}$ & & \\
\hline
\end{tabular}




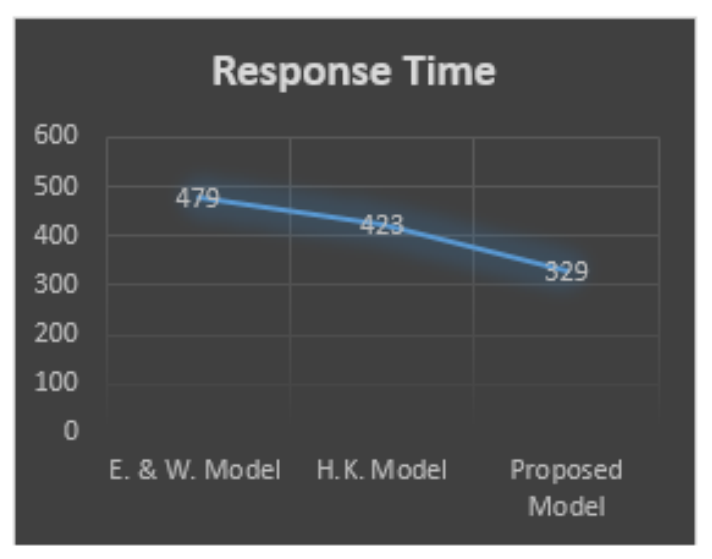

Figure 3. Comparison of Response Time of Example 1

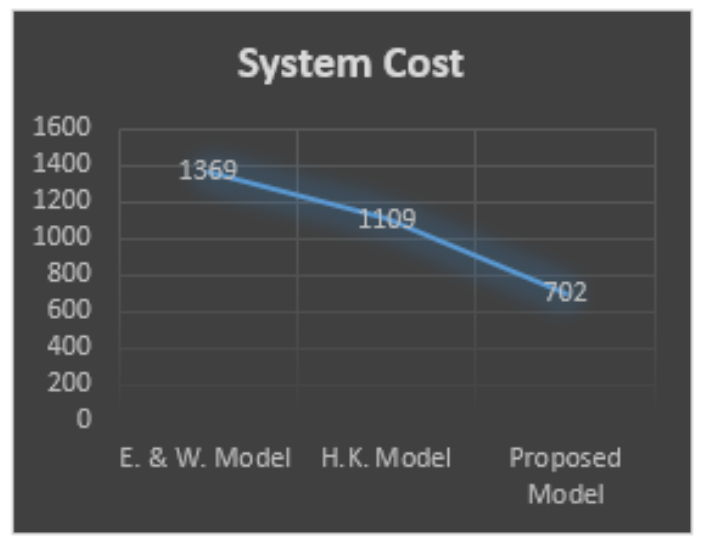

Figure 4. Comparison of system cost of example 1

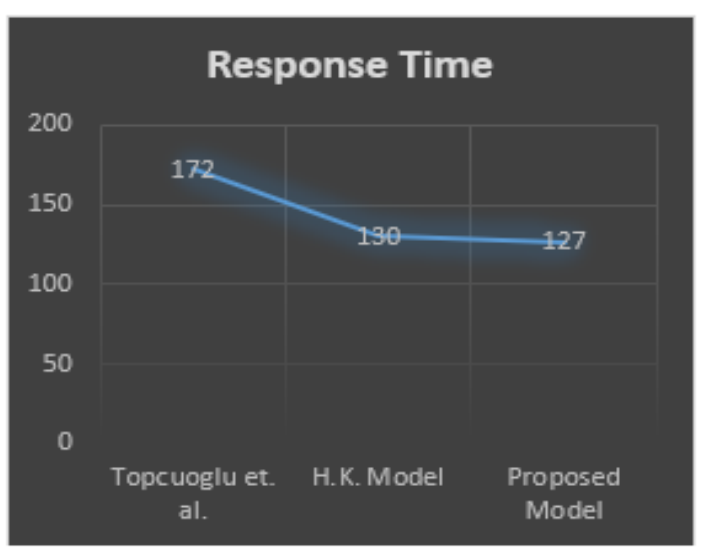

Figure 5. Comparison of response time of example 2

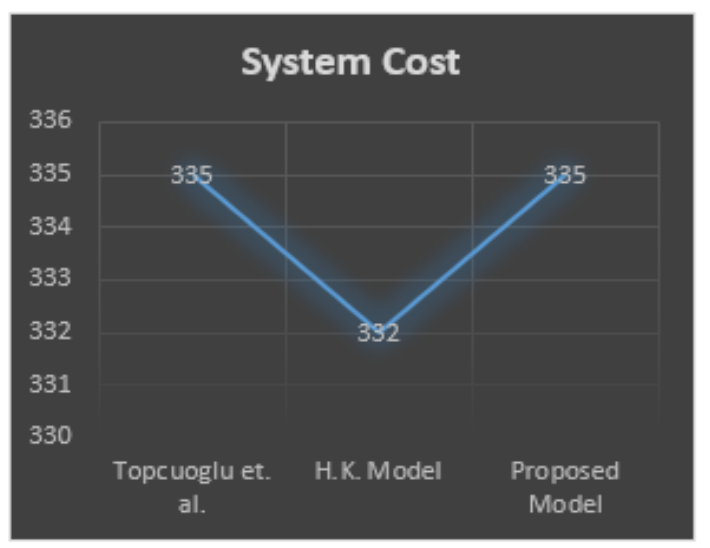

Figure 6. Comparison of system cost of example 2

\section{CONCLUSION AND FUTURE SCOPE}

In this paper a task allocation problem has been formulated and shown in the form of mathematical model. Paper proposes a novel algorithm for allocating the tasks on different processors with the objective of minimum response time and system cost by taking Execution Time and Inter Task Communication Time into consideration. The algorithm uses fuzzy $\mathrm{C}$ - means clustering technique (to form the clusters) and Hungarian method (for allocation of clustered tasks to different processors). Paper illustrated two scenarios for testing the proposed algorithm which gives optimum OPRT and system cost. The model has potential to minimize the Overall Process Response Time and System Cost (for overlapped data) by assigning an approximate balanced load to the processors as per literature studied. The limitation of paper is that it has a restriction of using for static load balancing and task assignment. Moreover, in the proposed clustering technique the number of iterations increases if the termination criterion is lowered, thus making the technique lengthy. Although the model presented is efficient enough for unsupervised data but leaves a number of situations where further work can be done by making use of flexibility of the clustering technique used. In future it can be further explored by varying the values of the parameters, of the clustering technique used, for static and dynamic systems.

\section{ACKNOWLEDGMENT}

The author is extremely grateful to Dr. Jogendra Kumar, Dr. Garima Verma and Dr. Fateh Singh for their kind support, valuable suggestions, comments and help.

\section{REFERENCES}

[1] Waraich, S.S. (2008). Classification of Dynamic Load Balancing strategies in a network of workstations. Fifth International Conference on Information Technology, New Generations, Las Vegas, NV, USA, pp. 1263-1265. https://doi.org/10.1109/ITNG.2008.166

[2] Huang, M.C., Hosseini, S.H., Vairaven, K. (2003). A Receiver-Initiated load balancing method in computer networks using fuzzy logic control. GLOBECOM '03. IEEE Global Telecommunications Conference (IEEE Cat. No.03CH37489), San Francisco, CA, USA, pp. 4028-4033. https://doi.org/10.1109/GLOCOM.2003.1258985

[3] Ahn, H.C., Youn, H.Y., Jeon, K.Y., Lee, K.S. (2007). Dynamic load balancing for large scale distributed system with intelligent fuzzy controller. IEEE International Conference on Information Reuse and Integration, Las Vegas, IL, USA, pp. 576-581. https://doi.org/10.1109/IRI.2007.4296682

[4] Zomaya, A.Y., Teh., Y.H. (2001). Observations on using Genetic algorithms for dynamic load balancing. IEEE Transaction on Parallel and Distributed Systems, 12(9): 899-911. https://doi.org/10.1109/71.954620

[5] Sriramdas, V., Chaurvedi, S.K., Gargama, H. (2014). Fuzzy arithmetic based reliability allocation approach during early design \& development. Expert Systems with Applications, 41(7): 3444-3449. https://doi.org/10.1016/j.eswa.2013.10.048

[6] Neelkantan, P., Sreekanth, S. (2016). Task allocation in 
distributed systems. Indian Journal of Science \& Technology, $9(31)$ : $1-10$ http://dx.doi.org/10.17485/ijst/2016/v9i31/89615

[7] Attiya, G., Hamam, Y. (2006). Task Allocation for maximizing reliability of distributed systems: A simulated annealing approach. Journal of Parallel and Distributed Computing, 66(10): 1259-1266. http://dx.doi.org/10.1016/j.jpdc.2006.06.006

[8] Kumar, H. (2015). A Heiristic model for task scheduling in heterogeneous distributed real time system under fuzzy environment. International Journal of Computer Applications, 111(2): 35-43.

[9] Hamed, A.Y. (2012). Task allocation for maximizing reliability of distributed computing systems using genetic algorithm. International Journal of Computer Networks and Wireless Communications, 2(5): 560-569.

[10] Topcuoglu, H., Hariri, S., Wu, M.Y. (2002). Performance effective \& low complexity task scheduling for heterogeneous computing. IEEE Transactions on Parallel and Distributed Computing, 13(3): 260-274. http://dx.doi.org/10.1109/71.993206

[11] Falta, G.D., Blasa, F., Cafiero, S., Fortina, G. (2013). Fault tolerant decentralized k-means clustering for asynchronous large scale network. Journal of Parallel and Distributed Computing, 3(3): 317-329. https://doi.org/10.1016/j.jpdc.2012.09.009

[12] Akbari, M., Rashidi, H. (2016). A multi objective scheduling algorithm based on cuckoo optimization for task allocation problem at compile time in heterogeneous systems. Expert Systems with Applications, 60: 234-248. https://doi.org/10.1016/j.eswa.2016.05.014

[13] Bahmani, A., Mueller, F. (2016). Efficient clustering for ultra scale application tracing. Journal of Parallel \& Distributed Computing, 98: 25-39. https://doi.org/10.1016/j.jpdc.2016.08.001
[14] Vidyarthi, D.P., Tripathi, A.K. (2001). Maximizing reliability of distributed computing systems with task allocation using simple genetic algorithm. J. System Architecture, 47(6): $549-554$ https://doi.org/10.1016/S1383-7621(01)00013-3

[15] Chu, W.W., Holloway, I.J., Lan, M.T., Efe, K. (1980). Task allocation in distributed data processing. Journal Computer, 13(11): 57-69. http://dx.doi.org/10.1109/MC.1980.1653419

[16] Kumar, H., Chauhan, N.K., Yadav, P.K. (2018). A high performance model of task allocation in distributed computing system using k-means clustering technique. International Journal of Distributed Systems \& Technologies, 9(3): 1-23. https://doi.org/10.4018/IJDST.2018070101

[17] Srinivasan, A., Geetharamani, G. (2013). Method for solving fuzzy assignment problem. Applied Mathematical Sciences, 7(113): 5607-5619. http://dx.doi.org/10.12988/ams.2013.37381

[18] Qinma, K., Hong, H., Jun, W. (2013). An effective iterated greedy algorithm for reliability-oriented task allocation in distributed computing systems. Journal of Parallel and Distributed Computing, 73(8): 1106-1115. https://doi.org/10.1016/j.jpdc.2013.03.008

[19] Rehman, S., Javaid, N., Rasheed, S., Hassan, K., Zafar, F., Naeem, M. (2018). Min-min scheduling algorithm for efficient resource distribution using cloud and fog in smart buildings. Proceedings of $13^{\text {th }}$ International conference on Broadband and Wireless Computing Communication and Applications, pp. 15-27. http://dx.doi.org/10.1007/978-3-030-02613-4_2

[20] Jang, S.H., Kim, T.Y., Kim, J.K., Lee, J.S. (2012). The study of genetic algorithm-based task scheduling for cloud computing. International Journal of Control and Automation, 5(4): 157-162. 